Palavras chave:

Espécies tropicais Composição química Taninos

Armazenamento de bebidas

Histórico:

Recebido 06/07/2012 Aceito 02/02/2015

Keywords:

Tropical species

Chemical composition

Tannins

Storing liquor

Correspondência: jonnys_33@hotmail.com

$\mathrm{DOl}$
Jonnys Paz Castro', Deise Morone Perígolo², Maria Lúcia Bianchi', Fábio Akira Mori', Alessandra de Souza Fonseca', Isabel Cristina Nogueira Alves', Francisco José de Vasconcellos ${ }^{3}$

\section{USO DE ESPÉCIES AMAZÔNICAS PARA ENVELHECIMENTO DE BEBIDAS DESTILADAS: ANÁLISES FÍSICA E QUÍMICA DA MADEIRA}

RESUMO: O processo de armazenamento de bebidas destiladas em barris de madeira é uma prática que visa à melhoria das características sensoriais da bebida, tais como cor, aroma e sabor. A qualidade da bebida destilada armazenada nesses barris depende de características da madeira como densidade, permeabilidade, composição química e anatomia, além do tipo de tratamento térmico dado à madeira durante a confecção dos barris. O Brasil possui uma grande diversidade florestal, principalmente no norte do país, na Amazônia. Essa região abriga milhares de espécies de árvores, porém, limita-se à utilização de pouco mais de duas espécies nativas e outras exóticas para o armazenamento de bebidas. Assim, objetivou-se, neste trabalho, determinar algumas das características físicas e químicas das madeiras de quatro espécies florestais da Região Amazônica. Os resultados obtidos para as espécies arbóreas aqui estudadas serão comparados com os de madeiras já utilizadas para o armazenamento de bebidas destiladas. As espécies estudadas foram angelim-pedra (Hymenolobium petraeum Ducke), cumarurana (Dipteryx polyphylla (Huber) Ducke), jatobá (Hymenaea courbaril L.) e louro-vermelho (Nectandra rubra (Mez) C.K. Allen). As árvores foram coletadas em área de manejo florestal da Empresa Precious Woods Amazon, em Silves, Amazonas. Dentre as análises realizadas foram determinados: teores de extrativos totais, quantidade de lignina, porcentagem de minerais (cinzas) e teores de taninos, além de densidade básica, análise elementar (CHNS-O) e análise térmica. Observou-se que a composição química estrutural (teores de lignina, holocelulose) e elementar (porcentagem de $\mathrm{C}, \mathrm{H}, \mathrm{N}$ e O) das madeiras possuem diferenças significativas. A madeira do jatobá possui maior teor de taninos e, na análise térmica, foi a madeira com menor perda de massa.

\section{USE OF AMAZONIAN SPECIES FOR AGING DISTILLED BEVERAGES: PHYSICAL AND CHEMICAL WOOD ANALYSIS}

ABSTRACT: The process of storing liquor in wooden barrels is a practice that aims to improve the sensory characteristics, such as color, aroma and flavor, of the beverage. The quality of the liquor stored in these barrels depends on wood characteristics such as density, permeability, chemical composition, anatomy, besides the wood heat treatment used to fabricate the barrels. Brazil has a great diversity of forests, mainly in the north, in the Amazon. This region is home to thousands of tree species, but is limited to the use of only a few native species to store liquors. The objective of this study was to determine some of the physical and chemical characteristics for four Amazon wood species. The results obtained in this study will be compared with others from woods that are traditionally used for liquor storage. The species studied were angelim-pedra (Hymenolobium petraeum Ducke) cumarurana (Dipteryx polyphylla (Huber) Ducke), jatobá (Hymenaea courbaril L.) and louro-vermelho (Nectandra rubra (Mez) CK Allen). The trees were collected from Precious Woods Amazon Company forest management area, in Silves, Amazonas. Analyzes such as: concentration of extractives, lignin amount, percentage of minerals (ash) and tannin content, density, elemental analysis (CHNS-O) and thermal analysis were done. It was observed that the chemical composition (lignin, holocellulose) and elemental analysis (percentage of $\mathrm{C}$, $\mathrm{H}, \mathrm{N}$ and $\mathrm{O}$ ) of the woods have significant differences. The jatobá wood presented higher tannin content, and in the thermal analysis, was that which had the lowest mass loss.

' Universidade Federal de Lavras - Lavras, Minas Gerais, Brasil

2 Universidade Federal de Minas Gerais - Belo Horizonte, Minas Gerais, Brasil

${ }^{3}$ Instituto Nacional de Pesquisas da Amazônia - Manaus, Amazonas, Brasil 


\section{INTRODUÇÃO}

Segundo o Instituto Brasileiro da Cachaça IBRAC (20I2), o Brasil possui capacidade instalada de produção de cachaça de mais de I,2 bilhão de litros por ano, gerando, aproximadamente, 600 mil empregos diretos e indiretos, o que evidencia a grande importância desse produto para a economia nacional.

A cachaça pode ser comercializada após a destilação ou passar por um período de armazenamento em barris de madeira, antes de ser engarrafada, o que agrega valor ao produto. $O$ processo de armazenamento de bebidas destiladas em barris de madeira é uma prática que visa à melhoria das características sensoriais da bebida, tais como cor, aroma e sabor. No Brasil, apesar do processo de armazenamento ser optativo ele é indispensável quando se deseja agregar qualidade e, consequentemente, valor a uma bebida destilada (MIRANDA et al., 2008). Por meio do armazenamento em barris de madeiras, podem-se corrigir eventuais defeitos da fermentação e da destilação, melhorando, assim, o paladar das bebidas destiladas (MORI et al., 2003).

A qualidade da bebida é influenciada pela espécie de madeira utilizada na confecção dos barris e, consequentemente, a qualidade da madeira utilizada é influenciada por uma série de variáveis como densidade, permeabilidade, composição química, anatomia etc. Essas características são capazes de alterar as propriedades gustativas e olfativas, bem como a cor das bebidas (LEÃO, 2006).

Atualmente, a madeira mais utilizada para o envelhecimento de bebida destilada é o carvalho (Quercus sp.). Depois do carvalho, no Brasil, a cerejeira (Amburana cearensis) e o bálsamo (Myroxylon peruiferum) são as espécies mais utilizadas para o armazenamento de bebida destilada.

O Brasil possui uma grande diversidade florestal, principalmente no Norte do País, na Amazônia. Essa região abriga, aproximadamente, 2,5 mil espécies arbóreas, cujas madeiras são diversificadas quanto à sua estrutura anatômica e propriedades tecnológicas (PAULA; ALVES, 1997). Porém, mesmo com todo esse potencial, limita-se à utilização de pouco mais de duas espécies nativas e outras exóticas para o armazenamento de bebidas. As espécies nativas, em sua maioria, possuem possibilidade de múltiplos usos, além de reunirem características favoráveis de adaptação às condições do ambiente, mas, para isso, é preciso conhecimento de seu potencial tecnológico (GOULART et al., 2012).
Portanto, o estudo de madeiras alternativas para a produçao de barris é uma necessidade. Nesse sentido, esse trabalho tem por objetivo obter informações sobre quatro espécies madeireiras brasileiras, fazendo a caracterização dessas espécies por meio de análises físicas e químicas e comparando alguns valores encontrados com os de espécies arbóreas já utilizadas para envelhecimento de bebidas (como o carvalho) ou já estudadas, anteriormente, para esse fim. Essas informações contribuirão positivamente para o desenvolvimento da indústria da tanoaria, pois a possibilidade de produção de barris para envelhecimento de bebidas, produzidos com madeiras da Amazônia, pode caracterizar um novo marco para a industrialização de certas espécies e uma nova fonte de matéria-prima para a confecção de barris, além da produção de bebidas diferenciadas.

\section{MATERIAL E MÉTODOS}

\section{Coleta do material}

As madeiras estudadas foram angelim-pedra (Hymenolobium petraeum Ducke), cumarurana (Dipteryx polyphylla (Huber) Ducke), jatobá (Hymenaea courbaril L.) e louro-vermelho (Nectandra rubra (Mez) C.K. Allen). As árvores de cada espécie foram coletadas na área de manejo localizada no município de Silves, Amazonas, na fazenda Monte Verde da Empresa Precious Woods Amazon. Foram coletados três indivíduos por espécie, exceto para Cumarurana, da qual foram coletados apenas dois indivíduos. De cada indivíduo foi retirado um disco de, aproximadamente, $3,0 \mathrm{~cm}$ de espessura na base de cada tora.

\section{Identificação das espécies}

A identificação foi realizada no Laboratório de Anatomia e Identificação da Madeira na Coordenação de Tecnologia e Inovação no Instituto Nacional de Pesquisas da Amazônia - COTI/INPA. Após a identificação, as amostras foram registradas na xiloteca - COTI/INPA.

\section{Caracterização do material}

Os discos foram subdivididos em cunhas. Foi destinado um par de cunhas opostas de cada disco para ensaios de determinação da densidade básica da madeira. Para as análises químicas, elementar, térmica e cromatográfica, foram separadas cunhas opostas para o preparo de amostras homogêneas. As cunhas opostas 
foram transformadas em maravalhas e, posteriormente, em serragem, com o auxílio de um moinho martelo e moinho tipo Wiley. As partículas obtidas foram classificadas em peneiras de 40, 60, 100, 200 e 270 mesh. As serragens obtidas em cada malha foram acondicionadas em sala climatizada à temperatura de $20^{\circ} \mathrm{C}$ e umidade relativa $60 \%$. As serragens de granulometria de 60 mesh foram utilizadas para a determinação de extrativos, minerais (cinzas) e lignina. Para a determinação do teor de taninos foram utilizadas as serragens de $60 \mathrm{e}$ 100 mesh. Para as análises térmica e elementar foram utilizadas amostras com granulometria de 270 mesh. Para as análises cromatográficas foi utilizado o material com granulometria entre 40 e 60 mesh.

\section{Determinação da densidade e análises química}

Para determinação da densidade básica, foi utilizada a norma da Associação Brasileira de Normas Técnicas - ABNT (2003). As análises químicas seguiram as normas M3/69, MII/77 e M70/7I da Associação Brasileira Técnica de Celulose e Papel - ABTCP (1974), para determinação de extrativos totais, minerais e lignina insolúvel, respectivamente. A percentagem de polissacarídeos (holocelulose) foi determinada por diferença, conforme a Equação I.

Holocelulose $(\%)=100$ - lignina $(\%)-$ extrativos $(\%)-$ minerais (\%)

A análise elementar foi realizada em um analisador elementar modelo Vario Microcube Elementar, seguindo a metodologia descrita por Paula et al. (20I I).

\section{Determinação do teor de taninos}

Foram preparados extratos, utilizando, aproximadamente, $200 \mathrm{mg}$ de madeira seca e triturada, amostras compostas, as quais foram extraídas com 10 $\mathrm{mL}$ de metanol $70 \%$, em decocção a frio e agitação constante por um período de 4 horas, segundo metodologia descrita por Castro et al. (2009). O extrato foi filtrado em papel filtro e o volume completado para $10 \mathrm{~mL}$ com metanol $70 \%$. O mesmo procedimento foi realizado com o solvente etanol a $70 \%$.

O doseamento de fenóis totais foi realizado, seguindo metodologia de Folin-Ciocalteau, com algumas modificações (WATERHOUSE, 2002). Os fenóis residuais foram determinados por complexação dos taninos por meio das ligações tanino-proteína, a proteína utilizada foi a caseína. $\bigcirc$ cálculo dos teores de taninos nas amostras foi determinado conforme a Equação 2, sendo T.T.(\%): Teor de Taninos; F.T.(\%): Fenóis Totais; F.R(\%): Fenóis Residuais.

T.T. $(\%)=$ F.T $(\%)-$ F.R. $(\%)$

\section{Análise térmica}

Para a análise térmica das madeiras, foram utilizadas amostras compostas, conforme descrito anteriormente. A análise de Termogravimetria/TG foi realizada em um aparelho Shimadzu-DTG 60AH. Foram utilizados, aproximadamente, $4 \mathrm{mg}$ de cada amostra, submetidas a uma taxa de aquecimento de $10^{\circ} \mathrm{C} \cdot \mathrm{min}^{-1}$, à temperatura inicial de $30^{\circ} \mathrm{C}$ até temperatura final de $550^{\circ} \mathrm{C}$, sob fluxo de nitrogênio $\left(50 \mathrm{~mL} \cdot \mathrm{min}^{-1}\right)$.

\section{Análises cromatográficas}

A hidrólise ácida para separação dos açúcares das madeiras foi realizada com ácido sulfúrico $72 \%$ em autoclave, por I hora, de acordo com as normas técnicas da ABTCP (1974). Os açúcares foram derivatizados, utilizando metodologia adaptada (PERÍGOLO, 20I4) baseada nos trabalhos de Dai et al. (20l0), Lv et al. (2009) e Strydom (1994). A identificação dos açúcares foi realizada por cromatografia líquida de alta eficiência (HPLC), em aparelho Shimadzu-Prominece, detector DAD (arranjo de diodos) injetor automático SIL - 20A, bombas LC-20AT, CBM-20A e DGU-20As e coluna Shim-pack VP-ODS (250 mm x 4,6 mm).

\section{Análises estatísticas}

Foram determinadas as estatísticas descritivas: média, desvio padrão e coeficiente de variação $\mathrm{C} . \mathrm{V}(\%)$. Os testes de Bartlett e Shapiro-Wilk foram realizados para verificar a homocedasticidade da variância e a normalidade da distribuição dos erros experimentais, respectivamente. O delineamento experimental utilizado foi o inteiramente casualizado - DIC, utilizando seis repetições e quatro tratamentos, para a determinação de densidade, análise química e elementar. Para a análise do teor de taninos, foi utilizado DIC, em esquema fatorial do tipo $4 \times 2 \times 2$ (espécies, granulometria e solvente), com três repetições. O teste paramétrico $\mathrm{F}$, foi utilizado a $5 \%$ de significância, assim como comparação múltipla das médias o teste ScottKnott, quando o teste $\mathrm{F}$ foi significativo. Todas as análises estatísticas foram realizadas, utilizando-se o software $R$ 2. I I.0 (R DEVELOPMENT CORE TEAM, 2008). 


\section{RESULTADOS E DISCUSSÃO}

\section{Densidade básica das madeiras}

A densidade básica é uma das propriedades mais importantes da madeira e seu valor depende da quantidade de tecido lenhoso por unidade de volume ou a quantidade de espaços vazios, assim como a presença de incrustações por goma-resina, cristais, sílicas, tilos e outros. A densidade da madeira está correlacionada à sua permeabilidade, já que madeiras de alta densidade apresentam volume menor de espaços vazios (MENDES et al., 200 I). Assim, a densidade e permeabilidade são características que devem ser observadas durante a confecção de barris para armazenamento de bebidas.

Os valores médios de densidade básica, desvio padrão e coeficiente de variação (C.V\%) obtidos para as espécies estudadas, são apresentados na Tabela I.

TABELA 1 Densidade básica média, desvio padrão e coeficiente de variação.

TABLE 1 Mean values of basic density, standard deviation and coefficient of variation.

\begin{tabular}{lccc}
\hline Espécies & $\begin{array}{c}\text { Densidade } \\
\left(\mathrm{g} \cdot \mathrm{cm}^{-3}\right)\end{array}$ & $\begin{array}{c}\text { Desvio } \\
\text { Padrão }\end{array}$ & $\begin{array}{c}\text { Coeficiente de } \\
\text { Variação }(\%)\end{array}$ \\
\hline Angelim-pedra & $0,615 \mathrm{C}^{1}$ & 0,015 & 2,459 \\
Cumarurana & $0,742 \mathrm{~B}$ & 0,009 & 1,207 \\
Jatobá & $0,789 \mathrm{~A}$ & 0,013 & 1,643 \\
Louro-vermelho & $0,535 \mathrm{D}$ & 0,006 & 1,200 \\
\hline
\end{tabular}

${ }^{1}$ Médias seguidas de letras maiúsculas iguais na coluna não diferem entre si estatisticamente pelo teste Scott-Knott em 5\% de probabilidade de erro.

Houve diferenças significativas de valores de densidade entre as espécies, sendo as madeiras de jatobá e louro-vermelho com maior e menor valor, respectivamente. Alcarde et al. (2010) observaram que a perda de volume do destilado, durante o período de envelhecimento, está diretamente relacionada à densidade da espécie de madeira utilizada nos tonéis.

$\mathrm{Em}$ aguardentes envelhecidas nos tonéis das madeiras de cerejeira (Amburana cearensis) com densidade de $0,60 \mathrm{~g} \cdot \mathrm{cm}^{-3}$, araruva (Centrolobium tomentosum) de $0,75 \mathrm{~g} \cdot \mathrm{cm}^{-3}$, jequitibá (Cariniana estrellensis) de $0,78 \mathrm{~g} \cdot \mathrm{cm}^{-3}$, cabreúva (Mycrocarpus frondosus) de $0,91 \mathrm{~g} \cdot \mathrm{cm}^{-3}$ e carvalho (Quersus sp) de $1,08 \mathrm{~g} \cdot \mathrm{cm}^{-3}$ as reduções médias de volume foram de 28 , 25, 24, 20 e 17\%, respectivamente. Quanto maior a densidade, menor a redução de volume. Assim, podemos inferir que as perdas de volume do destilado serão maiores para bebidas envelhecidas em barris fabricados com as madeiras de Louro vermelho e Angelim-pedra e menores para as madeiras de Cumarurana e Jatobá.

\section{Caracterização química das madeiras}

A quantidade da cada constituinte químico na madeira varia conforme a espécie, o que pode influenciar a composição final da bebida armazenada em barris produzidos com as diferentes espécie de madeira.

Os valores médios, desvio padrão e coeficiente de variação (C.V \%) da composição química estrutural das espécies estudadas são apresentados na Tabela 2.

Houve diferenças significativas quanto à quantidade dos componentes estruturais da parede celular presentes nas diferentes madeiras. As espécies possuem baixos valores de coeficiente de variação (C.V\%) para composição química, sendo que os maiores valores foram encontrados para os minerais, principalmente para a cumarurana e louro-vermelho.

Alguns valores médios de lignina, extrativos e minerais obtidos, neste trabalho, (Tabela 2) foram semelhantes àqueles encontrados por Santana e Okino

TABELA 2 Composição química das madeiras, desvio padrão, coeficiente de variação.

TABLE 2 Wood chemical composition, standard deviation, coefficient of variation.

\begin{tabular}{lcccc}
\hline \multirow{2}{*}{ Espécies } & \multicolumn{2}{c}{ Composição química (\%) } \\
\cline { 2 - 5 } Angelim-Pedra & Lignina & Extrativos & Minerais & Holocelulose \\
\hline \multirow{2}{*}{ Cumarurana } & $31,77 \mathrm{~B}^{1}$ & $11,52 \mathrm{~B}$ & $0,56 \mathrm{~A}$ & 56,15 \\
& $(0,55)^{*}(1,74)$ & $(1,03)(8,90)$ & $(0,05)(9,03)$ & - \\
\hline \multirow{2}{*}{ Jatobá } & $33,28 \mathrm{~A}$ & $9,56 \mathrm{C}$ & $0,40 \mathrm{~B}$ & 56,76 \\
& $(0,14)(0,42)$ & $(0,36)(3,80)$ & $(0,05)(12,58)$ & - \\
\hline \multirow{2}{*}{ Louro-vermelho } & $31,88 \mathrm{~B}$ & $12,78 \mathrm{~A}$ & $0,36 \mathrm{~B}$ & - \\
& $(0,44)(1,38)$ & $(0,52)(4,05)$ & $(0,02)(5,27)$ & 0,98 \\
& $33,63 \mathrm{~A}$ & $9,60 \mathrm{C}$ & $0,26 \mathrm{C}$ & 56,51 \\
\end{tabular}

${ }^{1}$ Médias seguidas de letras maiúsculas iguais nas colunas não diferem entre si estatisticamente pelo teste Scott-Knott em $5 \%$ de probabilidade de erro. *Valores entre parênteses são desvio padrão e coeficiente de variação respectivamente. 
(2007) em estudo realizado com as mesmas espécies, porém, com material obtido da Floresta Nacional de Tapajós, Santarém - PA (Tabela 3).

TABELA 3 Valores de referência para comparação da composição química das espécies.

TABLE 3 Reference values to compare the chemical composition of the species.

\begin{tabular}{lccc}
\hline Espécies & $\begin{array}{c}\text { Lignina } \\
(\%)\end{array}$ & $\begin{array}{c}\text { Extrativos } \\
(\%)\end{array}$ & $\begin{array}{c}\text { Minerais } \\
(\%)\end{array}$ \\
\hline Angelim-pedra $^{1}$ & 30,3 & 4,4 & 0,4 \\
Cumaru* $^{*}$ & 32,7 & 9,7 & 0,6 \\
Jatobá $^{1}$ & 30,3 & 8,5 & 0,4 \\
Jatobá $^{2}$ & 28,7 & 13,3 & 0,5 \\
Louro- vermelho $^{1}$ & 37,0 & 15,4 & 0,2 \\
Carvalho (Quersus sp) $^{2}$ & 25,9 & 10,5 & 0,5 \\
Carvalho(Quersus sp) $^{3}$ & 18,5 & 12,1 & -- \\
\hline
\end{tabular}

${ }^{1}$ Santana e Okino (2007). 'Cumaru (Diptery odorata). ${ }^{2}$ Mori et al. (2003). ${ }^{3}$ Leão (2006).

Houve algumas diferenças nos valores encontrados pelos autores citados, principalmente com relação aos extrativos. Tal diferença deve ter ocorrido em função da procedência do material coletado. Devese ressaltar que a cumarurana foi comparada com outra espécie do mesmo gênero, o cumaru (Dipteryx odorata). Para Santana e Okino (2007), essa variação pode ocorrer também pela idade das árvores coletadas.

O Carvalho é a madeira mais utilizada na fabricação de barris para envelhecimento de bebidas e representa um padrão de comparação. Os dados referentes aos teores de lignina obtidos por Leão (2006) e Mori (2003), apresentados na Tabela 3, são discordantes e possuem valores abaixo dos encontrados para todas as diferentes espécies estudadas nesse trabalho.
Os teores de alguns monossacarídeos presentes nas madeiras da Amazônia foram determinados. Para promover a quebra das ligações glicosídicas dos polissacarídeos para obtenção dos monossacarídeos, as amostras de madeira foram hidrolisadas com ácido. $\mathrm{Na}$ Tabela 4, apresenta-se o resultado das análises cromatográficas para determinação dos monossacarídeos.

TABELA 4 Porcentagem de alguns monossacarídeos presentes nas madeiras da Amazônia.

TABLE 4 Percentage of some monosaccharides present in the Amazonian woods.

\begin{tabular}{lcccc}
\hline Monossacarídeos & $\begin{array}{c}\text { Angelim- } \\
\text { pedra }\end{array}$ & Cumarurana & Jatobá & $\begin{array}{c}\text { Louro- } \\
\text { vermelho }\end{array}$ \\
\hline Glicose & 47,36 & 41,59 & 37,51 & 47,01 \\
Xilose & 5,90 & 10,84 & 14,15 & 5,69 \\
Manose & 1,40 & 1,13 & 1,39 & 2,28 \\
Ácido glucurônico & 1,02 & 1,87 & 1,35 & 1,08 \\
Galactose & 0,40 & 0,93 & 0,45 & 0,41 \\
Arabinose & $<0,50$ & $<0,50$ & $<0,50$ & $<0,50$ \\
\hline
\end{tabular}

Segundo Maga (1989), os compostos extraíveis da madeira originam-se dos componentes estruturais celulares da fração lignina e polissacarídeos. Destes, por hidrólise da fração celulose, origina-se a D-glicose, enquanto que da fração hemicelulose, são originados hexoses, pentoses, ácidos e grupos acetil. Além dos componentes estruturais, outros compostos solúveis e insolúveis da madeira ficam disponibilizados para extração.

$\mathrm{Na}$ Tabela 5, são apresentados os valores da análise elementar das madeiras amazônicas. Assim como para a composição química estrutural, os valores de porcentagem dos componentes elementares da madeira tiveram diferenças significativas, exceto para o teor de nitrogênio. $\mathrm{O}$ teor de $\mathrm{N}$ na madeira do angelim-pedra

TABELA 5 Composição elementar das madeiras, desvio padrão e coeficiente de variação.

TABLE 5 Wood elemental composition, standard deviation and coefficient of variation.

\begin{tabular}{lccccc}
\hline \multirow{2}{*}{ Espécies } & \multicolumn{5}{c}{ Elementos } \\
\cline { 2 - 6 } Angelim-Pedra & $\mathrm{C}(\%)$ & $\mathrm{H}(\%)$ & $\mathrm{N}(\%)$ & $\mathrm{S}(\%)$ & $\mathrm{O}^{* *}(\%)$ \\
\hline \multirow{2}{*}{ Cumarurana } & $47,87 \mathrm{~B}^{1}$ & $6,06 \mathrm{~B}$ & $1,16 \mathrm{~ns}$ & $0,043 \mathrm{~A}$ & 44,87 \\
& $(0,75)^{*}(1,56)$ & $(0,05)(0,80)$ & $(0,09)(7,55)$ & $(0,042)(77,37)$ & - \\
\hline \multirow{2}{*}{ Jatobá } & $48,45 \mathrm{~A}$ & $6,05 \mathrm{~B}$ & $1,03 \mathrm{~ns}$ & $0,023 \mathrm{~A}$ & 44,45 \\
& $(0,46)(0,95)$ & $(0,02)(0,25)$ & $(0,06)(5,49)$ & $(0,012)(40,35)$ & - \\
\hline \multirow{2}{*}{ Louro-vermelho } & $47,70 \mathrm{~B}$ & $5,84 \mathrm{C}$ & $1,02 \mathrm{~ns}$ & $0,012 \mathrm{~B}$ & 45,43 \\
& $(0,37)(0,78)$ & $(0,03)(0,57)$ & $(0,07)(6,41)$ & $(0,003)(26,21)$ & - \\
\hline
\end{tabular}

${ }^{1}$ Médias seguidas de letras maiúsculas iguais nas colunas não diferem entre si estatisticamente pelo teste Scott-Knott em $5 \%$ de probabilidade de erro. ns: não significativo em $5 \%$ de probabilidade de erro pelo Teste F. *Valores entre parênteses são desvio padrão e coeficiente de variação (\%) respectivamente. ${ }^{* *}$ Obtido por diferença. 
é um pouco superior aos normalmente encontrados nas literaturas, cujos valores podem variar de 0, I a I\%. Seye et al. (2003), estudando a cinética da biomassa, encontraram valores $48,06 \%$ de $\mathrm{C} ; 6,03 \%$ de $\mathrm{H} ; 0,70 \%$ de $\mathrm{N}$ e $45,21 \%$ de $\mathrm{O}$ para resíduos de madeiras. Os teores de enxofre $(\mathrm{S})$ encontrados para as espécies foram baixos, não ultrapassando $0,1 \%$. O teor de oxigênio foi obtido por diferença.

Paula et al. (20ll), analisando a composição elementar de diferentes resíduos lignocelulósicos encontraram valores de $0,1 \%$ de teor de nitrogênio para os resíduos de madeira. Os resultados encontrados, neste trabalho, para espécies amazônicas foram superiores aos encontrado por esses autores.

Segundo Munalula e Meincken (2009) o maior teor de nitrogênio está relacionado aos impactos ambientais e poluição do ar, em razão da formação de óxidos de nitrogênio e ácido nítrico

No caso de indivíduos da mesma espécie, a influência de fatores genéticos e da constituição do solo pode ser responsável por variações nos constituintes menores e nos componentes elementares das madeiras estudadas.

\section{Teor de taninos}

$\mathrm{Na}$ Figura I, ilustra-se a comparação das médias dos teores de taninos das espécies dentro de cada grupo variando-se a granulometria da amostra e o solvente utilizado. Médias seguidas de letras minúsculas iguais, dentro do mesmo grupo, não diferem entre

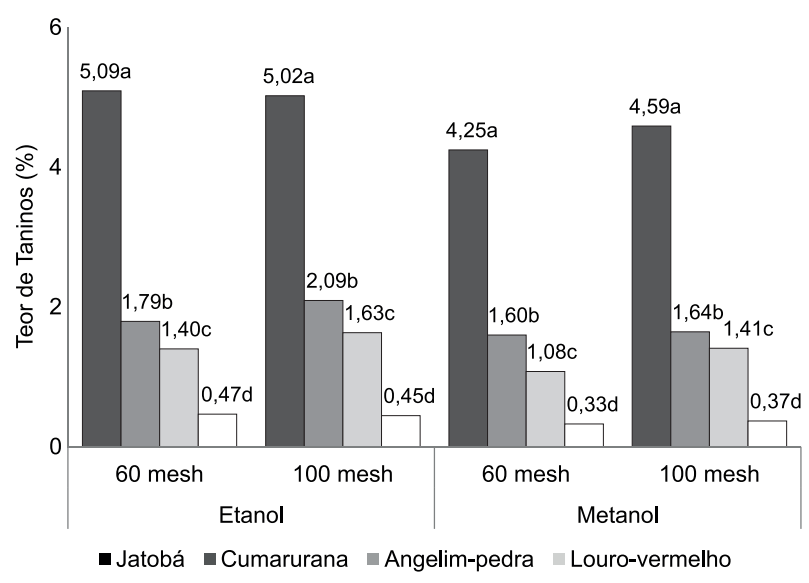

FIGURA 1 Teores de taninos das espécies nas diferentes granulometrias e solventes.

FIGURE 1 Tannin content of the species in different particle sizes and solvents. si estatisticamente pelo teste Scott-Knott em $5 \%$ de probabilidade de erro.

Os teores de taninos obtidos são estatisticamente diferentes entre as espécies, independente da granulometria ou solvente utilizado. Observa-se que a madeira do jatobá possui maiores teores de taninos, diferindo, significantemente, das demais madeiras. Já, a madeira do louro-vermelho possui o menor teor de taninos.

Os taninos são utilizados, principalmente, no curtimento de couro e também na produção de adesivos. $\mathrm{Na}$ indústria de bebidas, os taninos são um componente importante quando se trata das características sensoriais das bebidas como sabor, cor e aroma. Eles são extraídos dos barris de madeira onde as bebidas ficam armazenadas e são gradativamente hidrolisados durante o processo de maturação, formando ácido gálico e ácido elágico ou dímeros e oligômeros desses compostos (SILVA, 2006).

Assim, os taninos, como outros compostos oriundos da madeira favorecem o aumento da acidez da aguardente durante o processo de envelhecimento (ALCARDE et al., 2010). A legislação mantém elevado - limite máximo de acidez, visando a proteger a aguardente envelhecida, cuja acidez sempre aumenta com o decorrer do período de envelhecimento.

Taninos e compostos fenólicos, em geral, também estão envolvidos em reações com o cobre presente nos destilados, favorecendo o escurecimento da bebida (SINGLETON, 1995). No trabalho de Alcarde et al. (20l0), as aguardentes com maiores concentrações de compostos fenólicos foram também aquelas com menor concentração de cobre.

\section{Análise térmica}

A degradação dos componentes da madeira ocorre em três etapas distintas, na primeira etapa, ocorre perda da fração de hemiceluloses e parte da celulose e lignina. $\mathrm{Na}$ segunda etapa, ocorre degradação de celulose, e as hemiceluloses são convertidas no final dessa etapa. Na terceira etapa, ocorre a finalização da conversão da celulose e continuidade da degradação da lignina (ÓRFÃO et al., 1999).

$\mathrm{Na}$ Figura 2, representa-se a curva termogravimétrica das espécies estudadas. Em aproximadamente $40^{\circ} \mathrm{C}$ até $100^{\circ} \mathrm{C}$ ocorre perda de água do material. A porcentagem de umidade da madeira ficou em torno de 12,5\% (massa de água perdida). Entre 230 - $327{ }^{\circ} \mathrm{C}$ iniciou a degradação efetiva da madeira, tendo início a primeira etapa em que tem-se 
a degradação das hemiceluloses e parte da celulose e lignina. Segundo Alañón et al. (2010), a degradação das hemiceluloses e celulose produz alguns anidridos de monossacarídeos. Esses compostos foram encontrados apenas em madeiras de carvalho termotratadas e podem estar associados ao sabor levemente adocicado de algumas bebidas envelhecidas. $\mathrm{Na}$ terceira etapa, de $330{ }^{\circ} \mathrm{C}$ a $380{ }^{\circ} \mathrm{C}$ tem-se a degradação do restante da lignina e celulose.

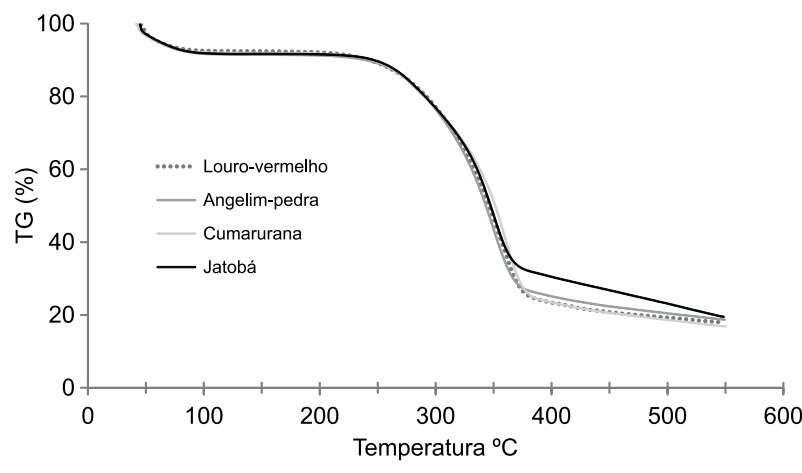

FIGURA 2 Curvas termogravimétricas das madeiras em atmosfera de nitrogênio.

FIGURE 2 Thermogravimetric curves of wood in a nitrogen atmosphere.

Observa-se que a $380^{\circ} \mathrm{C}$, a madeira de jatobá ainda possui aproximadamente $35 \%$ de sua massa, enquanto as outras espécies possuem aproximadamente $28 \%$. Isso pode significar diferenças na estrutura da lignina dessa madeira, já que, nessa temperatura todas as hemiceluloses e, praticamente, toda celulose já foram degradadas.

Os valores da fração sólida obtida da pirólise da madeira em atmosfera de $\mathrm{N}_{2}$ a $550^{\circ} \mathrm{C}$ encontrados foram de aproximadamente 19, 17, 19 e 18\% para angelim-pedra, cumarurana, jatobá e louro-vermelho, respectivamente.

É muito importante o conhecimento do perfil de degradação térmica da madeira que será utilizada na produção dos barris para armazenamento de bebidas. Isso, porque, durante a fabricação dos barris, a madeira deve ser aquecida para dar forma ao material e esse tratamento térmico altera quantitativa e qualitativamente a fração de substâncias extraíveis, durante a maturação de bebidas destiladas (FORLIN, 2005).

A aplicação de tratamentos térmicos na madeira, para posterior utilização na maturação de destilados, conduz à alteração do perfil sensorial das bebidas aumentando o aroma, coloração e corpo, diminuindo a pungência e a acidez das bebidas (CHATONNET et al., 1999; PUECH et al., 1984).

\section{CONCLUSÕES}

A madeira do jatobá possui maiores valores de densidade e maiores teores de taninos que as outras espécies, enquanto que a madeira de louro-vermelho possui a menor densidade e menor quantidade de taninos. O jatobá e o angelim-pedra possuem maiores teores de extrativos e minerais, respectivamente. A cumarurana foi a espécie com menor teor de extrativos, quantidade de taninos bem inferior à do jatobá e densidade próxima à densidade do jatobá. $\mathrm{Na}$ análise térmica das amostras foi observado que a madeira de jatobá possui menor perda de massa a $380^{\circ} \mathrm{C}$ em relação às outras espécies.

De acordo com os resultados obtidos, o jatobá seria a madeira mais indicada para fazer o armazenamento de bebidas. Ela possui a maior densidade e, com isso, possibilitaria uma menor perda de volume de líquido durante o armazenamento. Além disso, possui maiores teores de taninos. Entretanto, outros estudos são necessários para definir a melhor espécie. $O$ próximo passo será realizar as análises qualitativas e quantitativas dos extratos alcoólicos das madeiras estudadas.

\section{AGRADECIMENTOS}

Os autores agradecem à Fundação de Amparo à Pesquisa do Estado do Amazonas, pela bolsa de estudo concedida, à Fundação de Amparo à Pesquisa do Estado de Minas Gerais, à Empresa Precious Woods Amazon e à Coordenação de Tecnologia e Inovação no Instituto Nacional de Pesquisas da Amazônia.

\section{REFERÊNCIAS}

ALAÑÓN, M. E.; RUBIO, H.; DÍAZ-MAROTO, M. C.; PÉREZ-COELLO, M. S. Monosaccharide anhydrides, new markers of toasted oak wood used for ageing wines and distillates. Food Chemistry, London, v. II9, n. 2, p. 5055I2, Mar. 2010.

ALCARDE, A. R.; SOUZA, P. A.; BELLUCO, A. E. S. Aspectos da composição química e aceitação sensorial da aguardente de cana-de-açúcar envelhecida em tonéis de diferentes madeiras. Ciência e Tecnologia de Alimentos, Campinas, v. 30 , p. $226-232$, maio 2010 .

ASSOCIAÇÃO BRASILEIRA DE NORMAS TÉCNICAS. NBR I | 94 I: madeira: determinação da densidade básica. Rio de Janeiro, 2003. $6 \mathrm{p}$.

ASSOCIAÇÃO BRASILEIRA TÉCNICA DE CELULOSE E PAPEL. Normas técnicas ABCP. São Paulo, 1974. 
CASTRO, A. H. F.; PAIVA, R.; ALVARENGA, A. A. de; VITO, S. M. M. Calogênese e teores de fenóis e taninos totais em barbatimão [Stryphnodendron adstringens (Mart. ) Coville]. Ciência e Agrotecnologia, Lavras, v. 33, n. 2, p. 385-390, mar./abr. 2009

CHATONNET, P.; CUTZACH, I.; PONS, M.; DUBOURDIEU, D. Monitoring toasting intensity of barrels by chromatographic analysis of volatile compounds from toasted oak wood. Journal of Agricultural and Food Chemistry, Davis, v. 47, n. 10, p. 4310-4318, Oct. 1999.

DAI, J.; WU, Y.; CHEN, S. W.; ZHU, S.; YIN, H. P.; WANG, M.; TANG, J. Sugar compositional determination of polysaccharides from Dunaliella salina by modified RPHPLC method of precolumn derivatization with I-phenyl3-methyl-5-pyrazolone. Carbohydrate Polymers, Barking, v. 82, n. 3, p. 629-635, Oct. 2010.

FORLIN, F. J. Maturação de aguardente de cana composta com extrato de madeira de carvalho em embalagem de Polietileno tereftalato (PET). 2005. 182 p. Tese (Doutorado em Tecnologia de Alimentos) - Universidade Estadual de Campinas, Campinas, 2005.

GOULART, S. L.; MORI, F. A.; RIBEIRO, A. de O.; COUTO, A. M.; ARANTES, M. D. C.; MENDES, L. M. Análises químicas e da densidade básica da madeira de raiz, fuste e galho de barbatimão [(Stryphnodendron adstringens) Coville] de bioma Cerrado. Cerne, Lavras, v. I8, n. I, p. 59-66, jan./mar. 2012.

INSTITUTO BRASILEIRO DE CACHAÇA. Mercado interno. Disponível em: <http://www.ibrac.net/index. php?option $=$ com_content $\&$ view $=$ article\&id $=46 \&$ ltemid $=47>$. Acesso em: 10 jul. 2012 .

LEÃO, M. M. Influência do termotratamento na composição química da madeira de (Amburana cearensis), bálsamo (Myroxylon balsamum) e carvalho (Quercus sp.) e o impacto no aroma de uma modelo de cachaça. 2006. 86 p. Dissertação (Mestrado em Recursos Florestais) - Escola Superior de Agricultura "Luiz de Queiroz", Piracicaba, 2006.

LV, Y.; YANG, X.; ZHAO, Y.; RUAN, Y.; YANG, Y.; WANG, Z. Separation and quantification of component monosaccharides of the tea polysaccharides from Gynostemma pentaphyllum by HPLC with indirect UV detection. Food Chemistry, London, v. I I2, n. 3, p. 742746, Feb. 2009.

MAGA, J. A. The contribution of wood to the flavor of alcoholic beverages. Food Reviews International, London, v. 5, n. I, p. 39-99, 1989.

MENDES, L. M.; SILVA, J. R. M.; TRUGILHO, P. F. Influência da qualidade da madeira no envelhecimento de aguardentes. In: CARDOSO, M. G. (Ed.). Produção artesanal de aguardente. Lavras: UFLA, 200I. p. 19I-227.
MIRANDA, M. B.; MARTINS, N. G. S.; BELLUCO, E. de S.; HORII, J.; ALCARDE, A. R. Perfil físico-químico de aguardente durante envelhecimento em tonéis de carvalho. Ciência e Tecnologia de Alimentos, Campinas, v. 28, p. 84-89, dez. 2008. Suplemento.

MORI, F. A.; MENDES, L. M.; TRUGILHO, P. F.; CARDOSO, M. das $G$. Utilização de eucaliptos e de madeiras nativas no armazenamento da aguardente de cana-de-açúcar. Ciência Tecnologia de Alimentos, Campinas, v. 23, n. 3, p. 396400, set./dez. 2003.

MUNALULA, F; MEINCKEN, M. An evaluation of South African fuelwood with regards to calorifi $c$ value and environmental impact. Biomass and Bioenergy, Cambridge, v. 33, n. 3, p. 4I5-420, Mar. 2009.

ÓRFÃO, J. J. M.; ANTUNES, F. J. A.; FIGUEIREDO, J. L. Pyrolysis kinetics of lignocellulosic materials: three independent reactions model. Fuel, v. 78, p. 349-358, 1999.

PAULA, J. E. de; ALVES, J. L. H. de. Madeiras nativas: anatomia, dendrologia, dendrometria, produção e uso. Brasília: Fundação Mokiti Okada, 1997. 544 p.

PAULA, L. E. R.; TRUGILHO, P. F.; NAPOLI, A.; BIANCHI, M. L. Characterization of residues from plant biomass for use in energy generation. Cerne, Lavras, v. I7, n. 2, p. 237-246, abr./jun. 2011 .

PERÍGOLO, D. M. Quantificação de monossacarídeos de madeiras e polpas celulósicas por HPLC/DAD utilizando derivatização com I-fenil-3-metil-5pirazolona (PMP). 20I4. 8I p. Dissertação (Mestrado em Agroquímica) - Universidade Federal de Lavras, Lavras, 2014.

PUECH, J. L.; LEAUTÉ, R.; CLOT, G.; NOMDEDEU, L.; MONDIÉS, H. Évolution de divers constituants volatils et phenoliques des eaux-de-vie de cognac au cours de leur vieillissement. Sciences des Aliments, Paris, v. 4, p. 6580,1984

R DEVELOPMENT CORE TEAM. R: language and environment for statistical computing. Vienna: R Foundation for Statistical Computing, 2008. Disponível em: <http://www.R-project. org >. Acesso em: I5 jan. 2010.

SANTANA, M. A. E.; OKINO, E. Y. A. Chemical composition of 36 Brazilian Amazon forest wood species. Holzforschung, Berlin, v. 6I, p. 469-477, 2007.

SEYE, O.; CORTEZ, L. A. B.; GOMEZ, E. O. Estudo cinético da biomassa a partir de resultados termogravimétricos. In: ENCONTRO DE ENERGIA NO MEIO RURAL, 3., 2003, Campinas. Anais... Campinas, 2003. Disponível em: <http://www.proceedings.scielo.br/scielo.php?scrip$\mathrm{t}=\mathrm{sci}$ arttext\&pid =MSC0000000022000000200022\&ln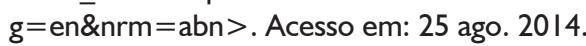 
SILVA, A. A. Compostos fenólicos em madeiras brasileiras. 2006. 130 p. Dissertação (Mestrado em Química Analítica) - Universidade de São Paulo, São Carlos, 2006.

SINGLETON, V. L. Maturation of wines and spirits: comparisons, facts, and hypotheses. American Journal of Enology and Viticulture, Davis, v. 46, n. I, p. 98- I I5, 1995.
STRYDOM, D. J. Chromatographic separation of I-phenyl-3methyl-5pyrazolone-derivatized neutral, acidic and basic aldoses. Journal of Chromatography A, Amsterdam, v. 678, p. 17-23, 1994.

WATERHOUSE, A. L. Determination of total phenolics. In: WROLSTAD, R. E. (Ed.). Current protocols in food analytical chemistry. New York: J. Wiley, 2002. p. I-4. 
